\title{
Análise do Aprendizado de Suporte Básico de Vida e a Obtenção Prévia da Autorização para Conduzir Veículos
}

\section{Basic Support of Life Learning Analysis and Previous Obtaining Authorization to Drive}

\author{
João Paulo Giliolia; Renan Verdinasse de Freitas ${ }^{\mathrm{a}}$; Felipe Labaki Pavarino ${ }^{\mathrm{b}}$; Henrique Parisotto Marson ${ }^{\mathrm{b}}$; \\ Nicézia Vilela Junqueira Franqueiro ${ }^{\mathrm{c}}$; Ricardo Alessandro Teixeira Gonsagad* \\ âniversidade Camilo Castelo Branco, Curso de Medicina. Fernandópolis/SP. \\ ${ }^{b}$ Faculdades Integradas Padre Albino, Curso de Medicina, Catanduva/SP. \\ 'Universidade Camilo Castelo Branco, Programa de Pós-Graduação Stricto Sensu em Engenharia Biomédica. Fernandópolis/SP.
}

dUniversidade Estadual de Campinas, Programa de Pós-Graduação em Ciências da Cirurgia, e Faculdades Integradas Padre Albino. Catanduva/SP.

*E-mail: novo02@uol.com.br

Recebido em: 31/08/2015; Aceito em: 06/11/2015

\section{Resumo}

O conhecimento teórico e as habilidades práticas durante o Suporte Básico de Vida (SBV) estão entre os determinantes mais importantes das taxas de sucesso em situações de emergência, o que ressalta a importância de dedicadas ações educacionais. O objetivo deste estudo foi analisar o aprendizado de acadêmicos do primeiro período de Medicina no treinamento prático do SBV, em imediato e médio prazo e correlacionar com a obtenção prévia da Carteira Nacional de Habilitação $(\mathrm{CNH})$. Método: estudo prospectivo no qual os dados foram obtidos em três momentos, a saber: um questionário (pré-teste) antes do treinamento em SBV, outro, imediatamente após e o último, 21 dias depois. O questionário continha dados demográficos e 15 questões de múltipla escolha sobre o assunto. Foram avaliados 79 alunos, com média de idade de 19,9 ( \pm $2,3)$ anos. Apenas quatro $(5,1 \%)$ haviam recebido treinamento prévio nos moldes do SBV. A média de acertos no primeiro teste foi de $29,7 \%$. $\mathrm{Na}$ avaliação pós-curso, a média de acertos foi $94,1 \%$. Já no terceiro teste, a média de acertos foi de $83,3 \%$. Utilizando o teste t Student para amostras independentes, não houve diferenças estatísticas entre aqueles que portavam e os que não portavam a $\mathrm{CNH}(\mathrm{p}=0,3264)$. Com base na caracterização dos alunos e do desempenho deles nas avaliações, pode-se concluir que o treinamento de SBV demonstrou uma importante qualidade na retenção de aprendizado. Evidenciou-se que portadores da $\mathrm{CNH}$ não tiveram resultados estatisticamente superiores aos que não a possuíam quanto aos conhecimentos adquiridos previamente ao treinamento.

Palavras-chave: Educação Médica. Serviços Médicos de Emergência. Ressuscitação Cardiopulmonar.

\begin{abstract}
The theory and practical skills during Basic Life Support (BLS) are among the most important determinants of success rates during emergencies, which emphasizes the importance of dedicated educational activities. The objective of this study is analyze the learning skills of the first period medical academics in practical training of BLS from now and medium term and inquire about the prior achievement of National Driver's License. Method: prospective study in which data were obtained from three moments: a questionnaire (pre-test) done before the course, the other immediately after the course and the final, 21 days later. In the questionnaire there were students'data and 15 multiple choice questions about BLS. The total of 79 students were evaluated, with average of $19.88( \pm 2.28)$ years old. Only four (5.06\%) had received prior training in SBV models. The average rate in the first test was $29.7 \%$. In the immediate post-test, the average rate was $94.06 \%$. In the second post-test, the average rate was $83.33 \%$. Using the t student's test for independent samples, there were no statistical differences between the group that had the National Driver's License and those who did not $(p=0.3264)$. Based on the students characters and their performance on the tests, it can be concluded that the BLS training demonstrated an important quality of learning. It became clear that carriers of National Driver's License had no statistically better results than those who do not own it concerning the knowledge acquired before training.
\end{abstract}

Keywords: Education, Medical. Emergency Medical Services. Cardiopulmonary Resuscitation.

\section{Introdução}

O Suporte Básico de Vida - SBV é definido como a abordagem inicial da vítima, podendo ser realizada por leigos capacitados ou profissionais da saúde, abrangendo desobstrução das vias aéreas, ventilação e circulação artificiais ${ }^{1}$. Consiste, assim, em uma gama de conhecimentos teóricos e práticos de pequena e média complexidade que, quando aplicados de maneira correta, aumentam a sobrevida e a taxa de sobrevivência dos indivíduos que apresentem risco à vida em um ambiente comumente pré-hospitalar ${ }^{2}$. Compõese dos três primeiros elos da cadeia de sobrevivência: reconhecimento precoce da emergência e rápida contactação do serviço de saúde, ressuscitação imediata do paciente e sua posterior desfibrilação, quando necessária ${ }^{3}$. Pelo fato de constituir-se de diretrizes que podem ser aplicadas em ambiente pré-hospitalar e em momento anterior à chegada da equipe médica, o SBV pauta-se na orientação à ação do leigo ou dos profissionais da saúde nos primeiros atendimentos à vítima. Por outro lado, quando realizada por pessoa não qualificada e/ou de maneira incorreta, pode comprometer a reabilitação do doente ou lhe trazer sequelas a posteriori.

Para obter a Carteira Nacional de Habilitação, o cidadão deve ser maior de idade e ter capacidades médicas e psicotécnicas adequadas. Somado a isso, deve passar por capacitação teórico-prática não só sobre condução de 
veículos em diversas condições, mas também sobre noções de primeiros socorros e Suporte Básico de Vida ${ }^{4}$. Pode-se inferir, assim, que os portadores de CNH são classificados como leigos capacitados à realização das etapas extra-hospitalares do SBV 5 .

De acordo com dados do Departamento Nacional de Trânsito - Denatran, o Brasil contava, em setembro de 2014, com 85.323.532 veículos emplacados, sendo 42.189.032 só na Região Sudesté . Como há relação entre o número de veículos e o de condutores, pode-se dizer que há um enorme contingente de cidadãos preparados para iniciar uma abordagem efetiva e imediata em situações que representam - isoladamente ou mesmo quando associadas - importantes causas de mortalidade no Brasil: as causas externas e as doenças do aparelho circulatório.

No Brasil, nas últimas décadas, as causas externas têm se tornado as principais causas de mortes de maneira geral. Em 1980, correspondiam a $11 \%$ das mortes totais e, em 2005, já representavam $15 \%{ }^{7}$. Isso afeta, em muito, grande parte de nossa população economicamente ativa, além de acarretar em grandes gastos ao sistema de saúde ${ }^{7,8}$. No município de São Paulo, em 1960, as causas externas representavam 6,5\% das mortes totais. Já em 1999, apontavam 14,2\%, correspondendo à maior causa de morte na faixa etária dos 5 aos 49 anos e à segunda causa de morte em geral. A comparação entre esses dados revela que houve crescimento de $90 \%$ no coeficiente de mortalidade por causas externas. Os acidentes de trânsito, especificamente, correspondiam a 11,5/100.000 mortes em 1960 e 13,6/100.000 mortes em 19999.

Ainda observando as causas externas como motivo de óbitos, no ano 2000, essas foram o terceiro grupo mais frequente no estado do São Paulo, entre os capítulos da Classificação Internacional de Doenças - CID 10, com 33,5 mil óbitos, representando 14,1\% do total de mortes. Em 2010, essa taxa caiu para o quarto lugar entre os capítulos da CID, com 25 mil óbitos ou $9,5 \%$ do total ${ }^{10}$.

Já com relação às mortes por doenças do aparelho circulatório, o número total de óbitos foi de 229.699 no ano de 1990, com os maiores valores no estado de São Paulo (67.274). Pouco mais de uma década depois, o número de óbitos aumentou $46,4 \%$ em números absolutos, com 336.213 mortes em 2011; 81.182 só no estado de São Paulo (ainda líder no número de mortes). As causas circulatórias representaram, portanto, $30,69 \%$ de todas as mortes brasileiras no ano de $2011^{11}$.

A grosso modo, o ingresso na vida acadêmica coincide com a obtenção da Carteira Nacional de Habilitação. Sabese ainda que a população matriculada no curso superior se enquadra na principal faixa etária sujeita às causas externas de mortalidade e, além disso, também corresponde àqueles de quem se espera alguma ação eficaz de suporte básico de vida (principalmente quando estudante de alguma área da saúde).

Além disso, sabe-se que a ressuscitação cardiopulmonar
(RCP) moderna completou cinquenta anos de existência em 2010. Durante esses anos de história, protocolos criados e aperfeiçoados com ênfase na qualidade e na rapidez das manobras de ressuscitação possibilitaram salvar inúmeras vidas no mundo todo, desde que aplicados de maneira correta $^{12-14}$. Protocolos esses obrigatoriamente ensinados a todos que almejam a obtenção da $\mathrm{CNH}$.

$\mathrm{O}$ ensino do SBV, porém, tende a ser realizado de forma superficial e pouco efetiva. Isso porque todo curso teórico para obtenção da $\mathrm{CNH}$ tem duração de 45 horas/aula ${ }^{15}$. Dentro desse período são ministradas não só as teorias de SBV, mas também noções de mecânica e direção defensiva. Além disso, pesquisas recentes inferem que o ensino de RCP necessita de aulas teóricas e práticas em grupos reduzidos, pois um número maior do que 8 alunos por professor já tende à redução significativa da eficácia do aprendizado (houve redução de ações efetivas quanto maior o grupo de capacitação em que o estudante participou $)^{16}$. A população não médica, portanto, tende a obter conhecimentos pouco efetivos de SBV (pouca carga teórica e ínfima carga prática, comumente não ministrada por profissionais qualificados; grupos numerosos; falta de contato com a situação real ou novas capacitações, acarretando esquecimento do aprendizado $)^{17}$. Tais dados corroboram a importância do rápido reconhecimento das variáveis de ocorrência e aplicação de eficiente abordagem individualizada de acordo com o $\mathrm{SBV}^{18}$. É indubitável que a maior causa de parada cardiorrespiratória - PCR sejam as causas não traumáticas, como as cardiovasculares, e, normalmente, não presenciadas no trânsito. Todavia, a capacitação deficitária do novo portador da $\mathrm{CNH}$ pode, também, e em menor âmbito, refletir as altas taxas de mortalidade por causas externas evitáveis no Brasil e predizer sobre as mortes evitáveis de causas não traumáticas.

É importante, portanto, analisar a eficiência e aplicabilidade dos ensinamentos ministrados aos futuros condutores, uma vez que serão eles os responsáveis pela mudança almejada nos índices de mortalidade brasileira. Dá-se ênfase também à análise do ensino para futuros médicos e sua relação com aqueles que já obtiveram algum tipo de contato com o curso de primeiros socorros antes de cursarem o primeiro período da graduação.

Este artigo tem como objetivo analisar o aprendizado de acadêmicos do primeiro período do curso de Medicina no treinamento prático de suporte básico de vida, em imediato e médio prazo, abordando se há diferença para a aquisição prévia da CNH pelos analisados e questionando o curso nos moldes do SBV ministrado em sua obtenção.

\section{Material e Métodos}

Trata-se de uma pesquisa prospectiva, quantitativa, analítica, quase-experimental. Quase-experimentos são delineamentos de pesquisas que não têm distribuição aleatória dos sujeitos pelos tratamentos, nem grupos-controle. Ao 
invés disso, a comparação entre as condições de tratamento e não tratamento deve sempre ser realizada com grupos não equivalentes ou os mesmos sujeitos antes do tratamento ${ }^{19}$. O estudo foi realizado no campus de Fernandópolis do curso de Medicina da Universidade Camilo Castelo Branco em abril de 2014. A amostra compreendeu os alunos do primeiro período do curso de Medicina, totalizando 79 alunos. Os dados foram coletados em três fases. Na primeira etapa, foi aplicado um questionário construído pelos pesquisadores com 15 perguntas de múltipla escolha sobre manobras e situações preconizadas no Suporte Básico de Vida. Os questionários foram aplicados pelos pesquisadores ao público-alvo de forma presencial. A segunda etapa ocorreu no final do curso de SBV (aulas teóricas com profissional treinado nas dependências do campus de Fernandópolis, utilizando o material do Laboratório de Habilidades). A terceira etapa constituiu-se na aplicação do mesmo questionário usado na primeira fase, para comparação das questões acertadas antes e após o curso, no início do segundo período. Os dados foram analisados com auxílio do programa BioEstat 5.0. Por meio dele, foram calculadas médias, medianas e demais medidas de posição e variabilidade. As médias globais de acertos e a correlação entre portar $\mathrm{CNH}$ e o SBV foram comparadas por meio do Teste de t Student. A significância estatística foi verificada com nível de 0,05 .

Os aspectos éticos foram respeitados, conforme a Resolução $n^{\circ} 466 / 12$, sendo esclarecido o objetivo do estudo aos participantes. A autorização para realização das entrevistas com os discentes foi obtida por meio da assinatura do Termo de Consentimento Livre e Esclarecido pelos alunos, no qual constavam os objetivos da pesquisa, seu caráter voluntário e sigiloso, além do contato do pesquisador e do orientador para eventuais esclarecimentos. Esta pesquisa foi submetida à análise do Conselho de Ética em Pesquisa das Faculdades Integradas Padre Albino, sendo aprovada pelo parecer consubstanciado sob o número CAAE 29136714.4.0000.5430.

\section{Resultados e Discussão}

Os formulários foram aplicados em 79 alunos do primeiro período do curso de Medicina da Universidade Camilo Castelo Branco, em Fernandópolis/SP, sendo 43 (54,4\%) do sexo feminino e $36(45,6 \%)$ do sexo masculino. A idade mínima encontrada foi de 17 anos, com máxima de 32 anos e média de 19,9 anos $( \pm 2,3)$.

Quando questionados sobre cursos prévios de SBV, desconsiderando o obrigatório para ser habilitado para obtenção da $\mathrm{CNH}$, quatro alunos $(5,1 \%)$ informaram que haviam recebido algum tipo de treinamento prévio na área pré-hospitalar. Verificou-se, também, que 54 alunos $(68,4 \%)$ portavam a Carteira Nacional de Habilitação.

A média de acertos no primeiro teste foi de 29,7\%. Na avaliação pós-curso, a média de acertos foi 94,1\%. Já no teste realizado no início do semestre posterior, a média de acertos foi de $83,3 \%$.

Realizou-se o teste t Student para amostras independentes, no qual não houve diferenças estatísticas entre o grupo que teve o curso de primeiros socorros para retirar a $\mathrm{CNH}$ e os que não tiraram a habilitação $(\mathrm{p}=0,3264)$.

O sucesso da recuperação da vítima de parada cardiorrespiratória - PCR ou em qualquer situação de emergência está intimamente ligado à presença de alguém capacitado para iniciar as manobras de ressuscitação cardiopulmonar - RCP, tão logo seja constatada a sua ocorrência ${ }^{20,21}$. Quando a RCP é realizada de forma eficaz, as taxas de sobrevida chegam a $50 \%{ }^{21,22}$.

O curso preparatório é ministrado aos condutores de automóveis com a intenção de capacitá-los a um atendimento pré-hospitalar. Deve-se esperar, em teoria, que as notas dos alunos não se alterem estatisticamente após aplicação de novos cursos e testes no primeiro período de Medicina. Os resultados obtidos nesta pesquisa, todavia, alertam que essa afirmação não procede, uma vez que os resultados entre alunos portadores da $\mathrm{CNH}$ foram estatisticamente insignificantes quando comparados a alunos que não portavam o documento.

A educação da população é uma meta importante nos principais modelos de atendimento à parada cardíaca, uma vez que sua ocorrência se dá predominantemente fora das unidades de saúde. Diversos estudos demonstraram a eficácia da intervenção precoce. Todavia, os maiores desafios encontrados ao educar a população são o número de pessoas a serem treinadas, a eficácia do treinamento e os níveis de retenção do conhecimento transmitido durante o curso ${ }^{17,23}$.

Dessa maneira, coloca-se em questão a importância do curso de Suporte Básico de Vida (SBV) ministrado durante a obtenção da Carteira Nacional de Habilitação, a qualidade e a capacitação dos profissionais que o ministram. Ainda, evidencia-se os fatores aprendizado e conhecimento adquiridos pelos condutores, uma vez que a diferença apresentada no trabalho quanto ao conhecimento sobre o assunto entre os alunos portadores da $\mathrm{CNH}$ e os não portadores da $\mathrm{CNH}$ foi insignificante.

Estudo holandês de novembro de 2014 atesta que a retenção de conhecimento nos treinamentos de primeiros socorros e de SBV está intimamente ligada à prática clínica prévia. Demonstrou que um semestre de experiência clínica trouxe um impacto significativo na retenção de conhecimentos e habilidades práticas. Assim, enfatizam que treinamentos com simulações repetitivas são mais eficientes do que aulas teórico-práticas simples com profissionais de saúde e experiência prática prévia - atendimento pré-hospitalar, serviços de ambulância e salas de emergência, por exemplo e podem beneficiar o aprendizado das habilidades ${ }^{14,24}$.

Com base nessa informação, evidencia-se a importância de adotar, no currículo médico e na graduação de profissionais da saúde, as habilidades práticas no aprendizado dos graduandos. Toma-se nota que, para portadores da $\mathrm{CNH}$ ou 
não, o aprendizado terá mais valia quando ministrado durante um longo período de tempo e com repetidas simulações.

\section{Conclusão}

De acordo com os dados colhidos nas avaliações e no desempenho dos alunos diante dessas, pode-se concluir que o treinamento Suporte Básico de Vida demonstrou ser uma importante qualidade de aprendizado. $\mathrm{O}$ trabalho evidenciou que portadores da Carteira Nacional de Habilitação não tiveram resultados estatisticamente superiores aos que não possuíam o documento e, consequentemente, treinamento ministrado nas aulas preparatórias para obtenção da $\mathrm{CNH}$.

\section{Referências}

1. Pergola AM, Araújo IEM. O leigo em situação de emergência. Rev Esc Enferm USP (Online) 2008;42(4):769-76.

2. Whitaker IY, Gutiérrez MGR, Koizumi MS. Gravidade do trauma avaliada na fase pré-hospitalar. ABM Rev Assoc Med Bras 1988;44(2):111-9.

3. Partridge R, Tan Q, Silver A, Riley M, Geheb F, Raymond $\mathrm{R}$. Rhythm analysis and charging during chest compressions reduces compression pause time. Resuscitation 2015;90:1337.

4. BRASIL. Lei $n^{\circ} 9.503$, de 23 de setembro de 1997. Código de Trânsito Brasileiro. Portal da Legislação: Leis Ordinárias 2015. [acesso em 30 out 2015]. Disponível em http://www. planalto.gov.br/ccivil_03/Leis/L9503.htm.

5. Pergola AM, Araújo IEM. O leigo e o suporte básico de vida. Rev Esc Enferm USP 2009;43(2):335-42.

6. Mynaio MCS. Seis características das mortes violentas no Brasil. Rev Bras Estud Popul 2009;26(1):135-40.

7. Gawryszewski VP, Morita M, Hidalgo NT, Valencich DMO, Brumini R. A mortalidade por causas externas no estado de São Paulo em 2005. Bol Epidemiol (São Paulo) 2006;3(33). [acesso em 1 mar 2015]. Disponível em http://www.cve. saude.sp.gov.br/agencia/bepa33 causa.htm

8. Gawryszewski VP, Jorge MHPM. Mortalidade violenta no município de São Paulo nos últimos 40 anos. Rev Bras Epidemiol 2000;3(1/3):50-69.

9. Mendes, JDV. Mortalidade por causas externas no estado de São Paulo em 2010. Bol Eletr Grupo Téc Aval Inf Saúde 2012;4(13). [acesso em 1 mar 2015]. Disponível em http:// portal.saude.sp.gov.br/resources/ses/perfil/profissional-dasaude/destaques//gais_jornal_13.pdf

10. Denatran - Departamento Nacional de Trânsito. [acesso em 1 mar 2015]. Disponível em http://www.denatran.gov.br/frota. htm

11. Brasil. Ministério da Saúde. Sistema de Informações sobre Mortalidade. [acesso em 30 ago 2015]. Disponível em http:// tabnet.datasus.gov.br/cgi/tabcgi.exe?idb2012/c08.def

12. Field JM, Hazinski MF, Sayre MR, Chameides L, Schexnayder SM, Hemphill R, et al. Part 1: executive summary - 2010
American Heart Association guidelines for cardiopulmonary resuscitation and emergency cardiovascular care. Circulation 2010;122:S640-56.

13. Silva PO, Oliveira TGS, Marta CB, Francisco MTR, Martins ERC, Sampaio CEP. Os alunos do ensino médio e o conhecimento sobre o suporte básico de vida. Rev Enferm UERJ 2012;20:621-4.

14. Miotto HC, Camargos FRS, Ribeiro CV, Goulart EMA, Moreira MCV. Efeito na ressuscitação cardiopulmonar utilizando treinamento teórico versus treinamento teóricoprático. Arq Bras Cardiol 2010;95(3):328-31.

15. Detran - Secretaria de Desenvolvimento e Planejamento Regional. Departamento Estadual de Trânsito de São Paulo. Permissão para dirigir ( $1^{\text {a }}$ habilitação). [acesso em 1 mar 2015] Disponível em http://www.detran.sp.gov.br/ wps/portal/portaldetran/cidadao/habilitacao/fichaservico/ habilitacaoPermissao

16. Mahling M, Münch A, Schenk S, Volkert S, Rein A, Teichner $\mathrm{U}$, et al. Basic life support is effectively taught in groups of three, five and eight medical students: a prospective, randomized study. BMC Med Educ 2014;14(1):185.

17. Ribeiro LG, Germano R, Menezes PG, Schmidt A, Pazin Filho A. Estudantes de Medicina Ensinam Ressuscitação Cardiopulmonar a Alunos do Fundamental. Arq Bras Cardiol 2013;101(4):328-35. doi: http://dx.doi.org/10.5935/ abc. 20130165

18. Malvestio MAA, Sousa RMC. Sobrevivência após acidentes de trânsito: impacto das variáveis clínicas e pré-hospitalares. Rev Saúde Pública 2008;42(4):639-47.

19. Maia ER, Gonçalves Júnior J, Lima EP, Campos W, Jovino EM, Fernandes FF, et al. Conhecimentos em atenção préhospitalar e suporte básico de vida por estudantes recémingressos de medicina. Rev Bras Educ Méd 2014;38(1):5964.

20. Canesin MF, Cardoso LTQ, Soares AE, Moretti MA, Timerman S, Ramires JAF. Campanhas públicas de ressuscitação cardiopulmonar: uma necessidade real. Rev Soc Cardiol Estado de São Paulo 2001;11(2):512-8.

21. Oliveira RG, Gonzalez MM, Oliveira EN, Nishimura LS, Quilici AP, Abrão KC, et al. Compressões torácicas contínuas realizadas por leigos antes e após treinamento. Rev Soc Bras Clín Méd 2012;10(2):95-9.

22. Rea TD, Helbock M, Perry S, Garcia M, Cloyd D, Becker $\mathrm{L}$, et al. Increasing use of cardiopulmonary resuscitation during out-of hospital ventricular fibrillation arrest: survival implications of guideline changes. Circulation 2006; 114:2760-5.

23. Marconato AMP. Curso de primeiros socorros para candidatos à carteira nacional de habilitação. Tese [Doutorado em Enfermagem] - Universidade Estadual de Campinas; 2013.

24. Ruijter PA, Biersteker HA, Biert J, Van Goor H, Tan EC. Retention of first aid and basic life support skills in undergraduate medical students. Med Educ Online 2014;19:24841. 